\title{
Nanostructured Lipid Carriers Loaded with 17-a-Estradiol Accumulate into Hair Follicles
}

\author{
Jeanifer Caverzan, ${ }^{\oplus *, a}$ Marcelo B. de Jesus ${ }^{\circledR b}$ and Nelson Durán ${ }^{c, d}$ \\ ${ }^{a}$ Instituto de Química, Universidade Estadual de Campinas (Unicamp), \\ CP 6154, 13084-862 Campinas-SP, Brazil \\ ${ }^{b}$ Departamento de Bioquímica e Biologia Tecidual, Instituto de Biologia, \\ Universidade Estadual de Campinas (Unicamp), CP 6109, 13083-970 Campinas-SP, Brazil \\ ${ }^{c}$ Laboratório de Carcinogênese Urogenital e Imunoterapia, Departamento de Biologia Estrutural e Funcional, \\ Instituto de Biologia, Universidade Estadual de Campinas (Unicamp), 13083-970 Campinas-SP, Brazil \\ ${ }^{d}$ Núcleo de Nanomedicina (Nanomed), Universidade Federal do ABC (UFABC), \\ 09210-580 Santo André-SP, Brazil
}

\begin{abstract}
To improve 17- $\alpha$-estradiol targeting in alopecia treatment, a formulation of nanostructured lipid carrier (NLC) prepared by the sonication method was developed. The physicochemical characterization showed an average diameter of $96 \pm 15 \mathrm{~nm}$ and the average zeta potential of $-17 \pm 6 \mathrm{mV}$. In addition, the encapsulation efficiency (EE) of 17- $\alpha$-estradiol, assessed by ultra-performance liquid chromatography (UPLC) fluorescence, was $99.6 \pm 0.3 \%$. Physical stability of NLC stored at room temperature was followed for 42 days through several physicochemical parameters, such as diameter, zeta potential, $\mathrm{pH}$ and EE. No changes were observed in the physicochemical parameters during this period, demonstrating the potential of NLC as a delivery system of 17- $\alpha$-estradiol. NLC dermal penetration, investigated by Franz diffusion cell and confocal microscopy using rhodamine-labeled NLCs, showed an accumulation of NLC in the outer portions of the epidermis, precisely in the hair follicle. The NLCs distribution in the skin suggests that $17-\alpha$-estradiol targeting was improved.
\end{abstract}

Keywords: nanostructured lipid carrier, 17- $\alpha$-estradiol, skin, confocal fluorescence, hair, alopecia

\section{Introduction}

Alopecia appears among the most common chronic problems in dermatology, characterized by hair loss; it affects both men and women, regardless of age. It is estimated a total of 30.2 billion dollars spent annually in treatment. ${ }^{1}$ Human scalp hair is highly exposed and plays an important role in the human appearance and thus affecting social interactions. Alterations in hair appearance (e.g., hair loss) can impinge upon social and psychological status, causing a reduction in self-esteem and confidence, having severe impacts on the quality of life of those affected. ${ }^{2,3}$ Although the high prevalence of alopecia in both genders, the therapeutic options are very limited and can result in side effects such as, gynecomastia, irritation and increased heart rate. ${ }^{4,5}$ Therefore, the search

*e-mail: caverzan@gmail.com for new therapeutic alternatives is highly desired, so the association of nanotechnology with this type of treatment has been studied. ${ }^{5}$

In the last decade, nanoparticles delivery to the skin has been proposed, and an enormous growth of research in this area has been noticed. ${ }^{67}$ Owing to the tight and cohesive characteristics of deeper layers of the skin, few formulations have been reported to reach the dermal delivery of therapeutics; therefore, nanoparticles delivery has been more efficiently used as an alternative for local therapy. ${ }^{6,8}$ Alternatively, the more rational use of nanoparticles for skin delivery seems to be the use of nanoparticles capable of delivering their cargo inside the hair follicles, close to their target structures. ${ }^{9}$

In particular, for alopecia, nanostructured lipid carriers (NLCs) are one of the main alternatives that have been employed to improve drug delivery at the skin. Probably due to their capacity for high drug loading, modulated drug 
release, and low drug leakage, NLCs are being employed in alopecia treatment research. ${ }^{10,11}$ Using squalene, a lipid naturally produced to moisturize the skin, NLC were produced to improve the fusion with the sebum in hair follicles. The particles were loaded with diphencyprone to treat alopecia areata, a chronic inflammatory disorder. The NLC formulations achieved skin accumulation and controlled release of the drug. ${ }^{12}$ Other work ${ }^{13}$ performed the encapsulation of cyproterone acetate in NLC. This study demonstrated that NLC could be a promising topical novel drug delivery system for specific targeting of hair follicles and sebaceous glands to treat androgenic skin disorders such as acne and alopecia. ${ }^{13}$

Among the drugs used for the treatment of alopecia, the 17- $\alpha$-estradiol is encountered as a safe alternative and, as shown, was capable of decelerating and stabilizing hair loss. ${ }^{14,15}$ However, some concerns have been raised about $17-\alpha$-estradiol efficiency. Besides the biocompatibility of lipids, some oils (e.g., buriti oil) acts as an active substance. Several natural extracts of various plants have been used to promote hair growth such as Asiasari radix, Eclipta alba and essential oil of Chamaecyparis obtusa, Ziziphus jujuba and Sophora flavescens. ${ }^{16,17}$ Unsaturated fatty acids, such as linoleic acid and oleic acid, have demonstrated activity against hair loss by inhibiting the 5 - $\alpha$-reductase enzyme. These fatty acids are present in variable quantities in some edible plants such as Carthamus tinctorius L. (Saffron), Helianthus annuus L. (sunflower), Linum usitatissimum L. (linseed), Sorghum bicolor (L.) Moench (sorghum) and bran Oryza sativa L. (rice). ${ }^{16}$

Therefore, in light of the recent findings, a new NLC formulation was prepared to improve the $17-\alpha$-estradiol properties to enhance the delivery of the drug inside the hair follicles, close to its target structures. Then, this research aimed to use natural oil in the formulation of NLC for delivery of $17-\alpha$-estradiol and to evaluate their joint action on alopecia, expecting to enhance the drug delivery inside the hair follicles. By an increase of the 17- $\alpha$-estradiol efficiency, also, by a decrease of the drug washout, and with its sustained release, lower doses of 17 - $\alpha$-estradiol might be applied and improve the treatment of alopecia.

\section{Experimental}

\section{Materials}

Myristyl myristate (MM) was donated by Croda (Campinas, Brazil); $17-\alpha$-estradiol and Pluronic F68 were obtained from Sigma-Aldrich (Saint Louis, USA); buriti oil was donated by Inovam Brasil (Ji-Paraná, Brazil); ceramides derived from Sederma (Le Perray,
France); 1,2-dioleoyl-sn-glycero-3-phosphoethanolamine$N$-(lissamine 10 rhodamine B sulfonyl) (rhodamine-PE) was purchased from Avanti Polar Lipids (Alabaster, USA); and Alexa Fluor 488, and TO-PRO-3 were purchased from Invitrogen (Carlsbad, USA).

\section{NLC preparation}

Nanostructured lipid carriers were prepared using the sonication method. Briefly, the solid lipid (SL) was heated $10{ }^{\circ} \mathrm{C}$ above its melting temperature (i.e., $50{ }^{\circ} \mathrm{C}$ for $\mathrm{MM}$ ), then, a mixture of oils (buriti oil and ceramides) was added to SL. In the molten lipids mixture, 17 - $\alpha$-estradiol and Tween 80 were added, and this mixture added to a solution of the stabilizer Pluronic F68 and sonicated for $30 \mathrm{~min}$. After this process, the dispersion was cooled in an ice bath until the temperature of $25^{\circ} \mathrm{C}$ obtaining the NLCs. Labeled NLCs were prepared as described above, but with the addition in the lipid phase of the fluorescent marker-ammonium salt of rhodamine-PE at a concentration of $0.1 \%$ in the lipid molar-fraction.

\section{Stability studies}

Physical stability of the NLC was evaluated by their average diameter sizes, and, also, by $\mathrm{pH}$ measurements of their dispersions stored at $4{ }^{\circ} \mathrm{C}$ and room temperature. Also, methylparaben $(0,15 \%)$ and propylparaben $(0.05 \%)$ were added to the NLC dispersions to avoid contamination by microorganisms.

\section{Mean diameter (Z-average) and zeta potential}

The nanoparticle dispersion was diluted with deionized water $(1: 10 \mathrm{v} / \mathrm{v})$, then, the mean diameter (Z-average) and zeta potential were measured by the technique of photon correlation spectroscopy and electrophoretic mobility, respectively, using the equipment Nano ZS Malvern Zetasizer with a fixed angle of $173^{\circ}$ at $25^{\circ} \mathrm{C} .{ }^{18}$

\section{Encapsulation efficiency}

The encapsulation efficiency (EE) was determined by measuring the concentration of free $17-\alpha$-estradiol in the dispersion medium. For this, an aliquot of the NLC dispersion was transferred to centrifuge tubes containing Microcon filter coupled to an ultrafiltration membrane (10,000 MWCO, Millipore) for the retention of the particles. The tubes were centrifuged at $6,500 \mathrm{rpm}$ in an Eppendorf centrifuge for $5 \mathrm{~min}$ and the concentration of $17-\alpha$-estradiol was determined by liquid chromatography 
Table 1. Lipid composition of NLC

\begin{tabular}{lccccc}
\hline Formulation & $\begin{array}{c}\text { Tween } 80 / \\
\%(\mathrm{~m} / \mathrm{m})\end{array}$ & $\begin{array}{c}\text { Myristyl myristate / } \\
\%(\mathrm{~m} / \mathrm{m})\end{array}$ & $\begin{array}{c}\text { Pluronic F-68/ } \\
\%(\mathrm{~m} / \mathrm{m})\end{array}$ & $\begin{array}{c}\text { Buriti oil / } \\
\%(\mathrm{~m} / \mathrm{m})\end{array}$ & $\begin{array}{c}17-\alpha \text {-Estradiol / } \\
\%(\mathrm{~m} / \mathrm{m})\end{array}$ \\
\hline NLC0 & 1.3 & 2 & 1.5 & 0.14 & 0.000 \\
NLC1 & 1.3 & 2 & 1.5 & 0.14 & 0.025 \\
\hline
\end{tabular}

with ultra-performance, in an UPLC equipment (Waters Acquity) with a diode array detector and fluorescence. The analysis was performed at $25{ }^{\circ} \mathrm{C}$ on a $\mathrm{C} 18$ column (2.1 $\mathrm{mm} \times 50 \mathrm{~mm}$ UPLC Acquity, Waters) column with a pre-filter. The mobile phase was water:acetonitrile $(70: 30, \mathrm{v} / \mathrm{v})$ that was filtered through a $0.22 \mu \mathrm{m}$ membrane (Millipore). The isocratic flow rate was $0.2 \mathrm{~mL} \mathrm{~min}^{-1}$, and the detector was set at a wavelength of $312 \mathrm{~nm}$ for emission and excitation at $280 \mathrm{~nm} .{ }^{19}$

\section{Evaluation of skin permeation}

To simulate the application of a commercial product in the skin, NLC dispersions were mixed on Carbopol gel $(0.1 \%)$. To obtain the gel, Carbopol $(0.1 \mathrm{~g})$ was dissolved in $9.9 \mathrm{~mL}$ of distilled water, after homogenization, the $\mathrm{pH}$ was adjusted to 7.0 with triethanolamine. Propylene glycol $(0.5 \%, \mathrm{~m} / \mathrm{m})$ was added to the gel as the wetting agent.

Franz diffusion cells were prepared from human skin taken from the abdomen of women aged between 29 and 40 years, donated after plastic surgery (ethics committee permission No. 47823615.8.0000.5404). The receiver cell was filled with sodium phosphate buffer $\left(0.05 \mathrm{~mol} \mathrm{~L}^{-1} \mathrm{pH} 7.4\right)$ with Tween $80(5 \%)$. The skin was treated with the following formulations: labeled NLCs in Carbopol gel $(0.1 \%)$ and rhodamine-PE in Carbopol gel $(0.1 \%)$. The Franz cells set up in a tub with water at $32{ }^{\circ} \mathrm{C}$ under stirring. After $1 \mathrm{~h}$, the skin was removed from Franz cells, and skin blocks were subsequently frozen and cut on a cryostat (Leica CM1860). These sections ( $8 \mathrm{~mm}$ thickness) were collected on slides and fixed with methanol and acetone.

After fixation, the sections were washed 3 times with PBS (phosphate buffered saline). Then, the sections were permeabilized with Triton X-100 (0.2\% in PBS) for $5 \mathrm{~min}$. Actin filaments were labeled with Alexa Fluor 488-conjugated phalloidin and nuclei with TOPRO3; both incubated at room temperature for $30 \mathrm{~min}$. Then, samples were washed three times with PBS; coverslips were mounted on glass slides using Dako mounting medium (Dako, Carpinteria, USA). The samples were analyzed using a Leica TCS SP5 confocal microscope (Leica Microsystems, Wetzlar, Germany) equipped with a $60 \times$ oil immersion lens. Image-processing program
Las AF 2.6.0 7266Leica CMS Microsystems GmbH was used for capturing the images. The images were acquired using the pinholes set to one Airy unit for each channel in a sequential manner, and images were analyzed using ImageJ software. ${ }^{20}$

\section{Results and Discussion}

The NLC produced are particularly appealing for skin delivery application

Initially, the solubility of $17-\alpha$-estradiol in some lipids like lanolin and MM was tested. The 17- $\alpha$-estradiol was insoluble in most tested lipids compositions, except in MM containing Tween 80. Thus, NLCs produced from MM, buriti oil, Tween 80, and Pluronic F-68 were used to encapsulate 17- $\alpha$-estradiol (Table 1). Two formulations were prepared by the sonication method: the bare NLC (NLC0) and the formulation containing $0.025 \%$ of 17 - $\alpha$-estradiol (NLC1). The produced NLCs had diameters of around $100 \mathrm{~nm}$, a narrow size distribution (polydispersity index, PI, about 0.3$)$, and a negative zeta potential $(-17 \mathrm{mV})$. NLC1 showed a very high percentage of encapsulation of 17- $\alpha$-estradiol (EE 99\%). Importantly, even after the incorporation of virtually all the $17-\alpha$-estradiol, there was no alteration in the physicochemical properties of the NLC1 when compared to the NLC0 (Table 2).

Table 2. Physicochemical characteristics of NLC

\begin{tabular}{lccc}
\hline NLCs & $\begin{array}{c}\text { Mean } \\
\text { diameter } / \mathrm{nm}\end{array}$ & PI & $\begin{array}{c}\text { Zeta } \\
\text { potential } / \mathrm{mV}\end{array}$ \\
\hline NLC0 & $104 \pm 14$ & $0.3 \pm 0.1$ & $-16 \pm 2$ \\
NLC1 & $96 \pm 15$ & $0.3 \pm 0.1$ & $-17 \pm 6$ \\
\hline
\end{tabular}

NLC: nanostructured lipid carrier; PI: polydispersity index. The diameter and zeta potential of NLC0 and NLC1 diluted in water were measured using a Zetasizer Nano (Malvern Instruments Ltd). For particle lipid composition, see Table 1 . The statistic difference between NLC with and without active was of $p>0.05$ for diameter, PI and zeta potential compared through analysis of variance (ANOVA). Each value represents the mean \pm -standard deviation (S.D.) $(n=6)$.

The physicochemical characteristics, showed in Table 2, of the produced NLCs are particularly appealing for skin delivery application. The diameter of the NLCs were below $200 \mathrm{~nm}$, which is consistent with the hair follicle for topical 
application. ${ }^{21}$ In fact, smaller NLCs (ca. $100 \mathrm{~nm}$ ) have better dermal penetration compared to the bigger ones. ${ }^{22}$

Additionally, the PI value obtained was ca. 0.3. According to Parmar et al. ${ }^{23}$ and Septiyanti et al. ${ }^{24}$ values below 0.3 represent narrow size distribution. Once PI value obtained was ca. 0.3 , is possible to consider that particles have narrow size distribution, which means that the preparation conditions (i.e., sonication time and intensity) were appropriated. Narrow size distribution also contributed to particles stability because it prevents Ostwald aging..$^{25}$

The negative zeta potential $(-17 \pm 6 \mathrm{mV})$ found for the NLCs may contribute to skin penetration, as demonstrated for negatively charged liposomal formulations. ${ }^{26}$ This parameter is also related to the stability of colloidal systems. It is an indicator of the repulsion degree between nanoparticles with similar charges, and this repulsion avoids nanoparticle aggregation. ${ }^{27}$ According to Rigon et al. ${ }^{28}$ particles with zeta potential higher than $+30 \mathrm{mV}$ or lower than $-30 \mathrm{mV}$ are considered physically stable due to repulsion forces. Once the NLC zeta potential was under $-30 \mathrm{mV}$, its stability only can be explained by the use of non-ionic surfactant that, according to Pivetta et al. ${ }^{27}$ tends to maintain the formulation stable due to the steric stabilization.

Finally, the high EE achieved is likely related to the hydrophobicity of 17- $\alpha$-estradiol, which has a high affinity for lipids used. In addition, the incorporation of oil (i.e., caprylic/capric triglyceride) in the solid lipid matrix increases the space between the chains of the lipids, thereby reduces the expulsion of the drug during recrystallization process. ${ }^{29}$ High EE values (> 99\%) are observed even after 30 days of storage at $4{ }^{\circ} \mathrm{C}$ and room temperature $\left(25^{\circ} \mathrm{C}\right)$.

\section{NLCs stability}

After the physicochemical characterization of the NCLs, the next step was to assess their physicochemical stability over time. First, the diameters of the particles overtime at $4{ }^{\circ} \mathrm{C}$ and room temperature were analyzed. Both formulations (i.e., with or without $17-\alpha$-estradiol) kept their size stable over 7 weeks (Figures 1 and 2). Although we observed some increase in PI over time, during 14 days, the particles kept well their uniformity at $4{ }^{\circ} \mathrm{C}$ (Figure 2), while particles kept at room temperature (Figure 1) showed to be uniform during 35 days; for both conditions, the PI values remained below 0.4 .

Using the same surfactant (Pluronic F68), Chirio et al. ${ }^{30}$ developed solid lipid nanoparticles (SLN). Their stability study was performed during 30 day-storage, and it was monitored particle mean size and zeta potential over time by dynamic light scattering measurements. Similarly, they did not find significant differences in these parameters over time. This result indicates that the steric stabilization mechanism caused by this surfactant could contribute to particle stability.

Next, the $\mathrm{pH}$ values of formulations were evaluated. Initially, the formulations had their $\mathrm{pH}$ values around 6, precisely, at 6.3 for NLC0 and 6.4 for NLC1. There were no alterations in $\mathrm{pH}$ for any formulation for 7 weeks (Figure 3). These results indicate that the formulations preserved their physicochemical properties for a long period (7 weeks). It is particularly important because, according to Choi et al., ${ }^{31}$ the $\mathrm{pH}$ is among the potential factors which determines the stability and release properties of entrapped materials from lipids nanoparticles.

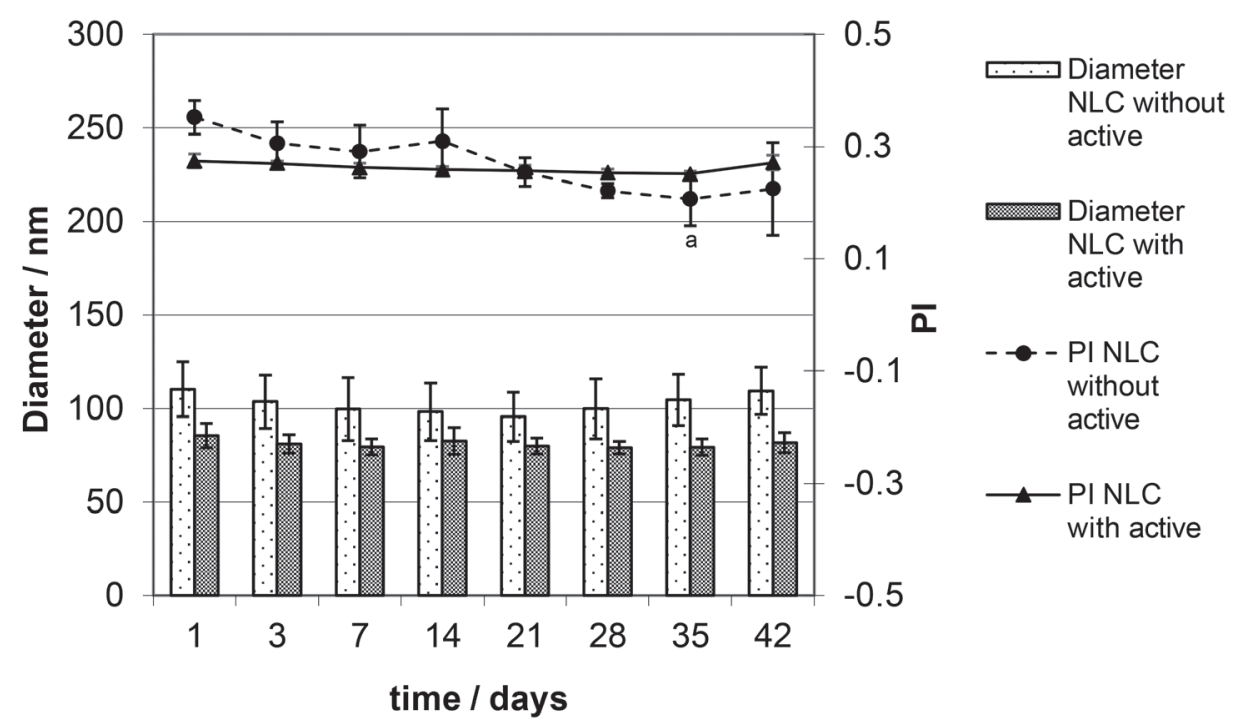

Figure 1. NLC diameter and size distribution (PI) of the formulations (stored at room temperature) as a function of time. a: Corresponds to a statistical difference $(p<0.05)$ in relation to the starting point $(1$ day) and analyzed through analysis of variance (ANOVA) with post-analysis using the Dunnett's test. Results presented as the mean and standard deviation of three independent experiments $(n=3)$. 

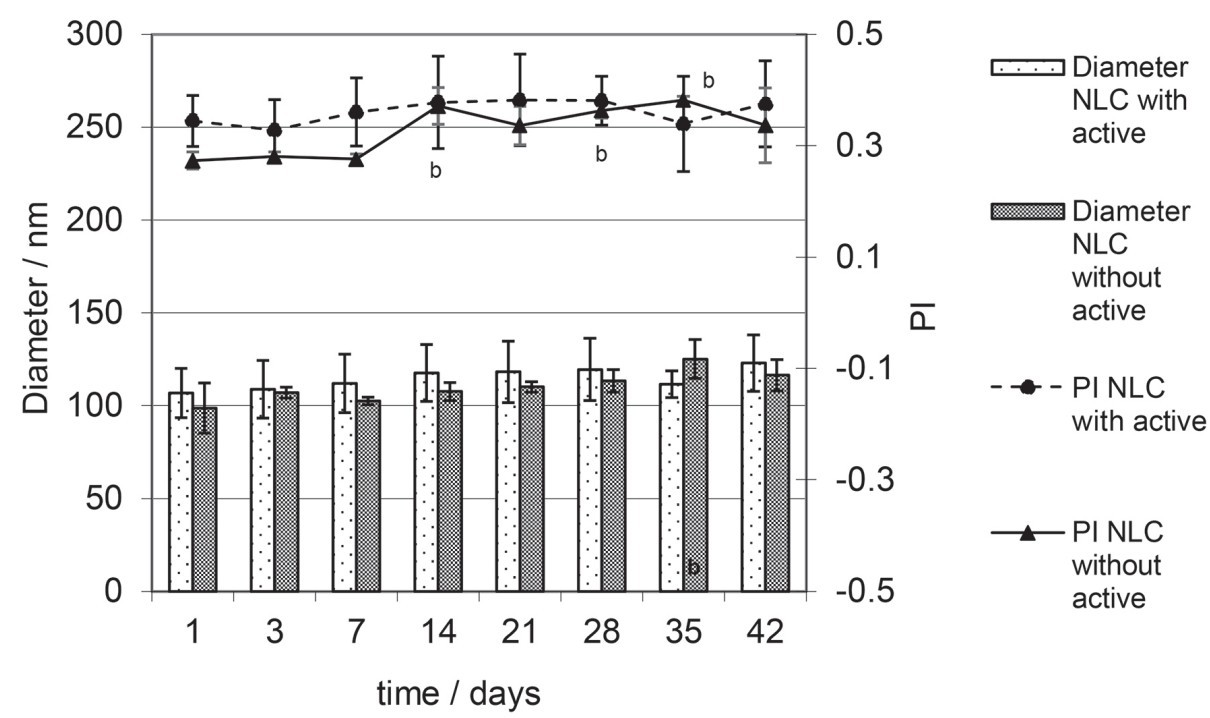

Figure 2. NLC diameter and size distribution (PI) of the formulations with and without active (stored in the refrigerator) versus time. b: Corresponds to the statistical difference $(p<0.01)$ in relation to the starting point ( 1 day) and analyzed through ANOVA with post-analysis using the Dunnett's test. Results presented as the mean and standard deviation of three independent experiments $(n=3)$.

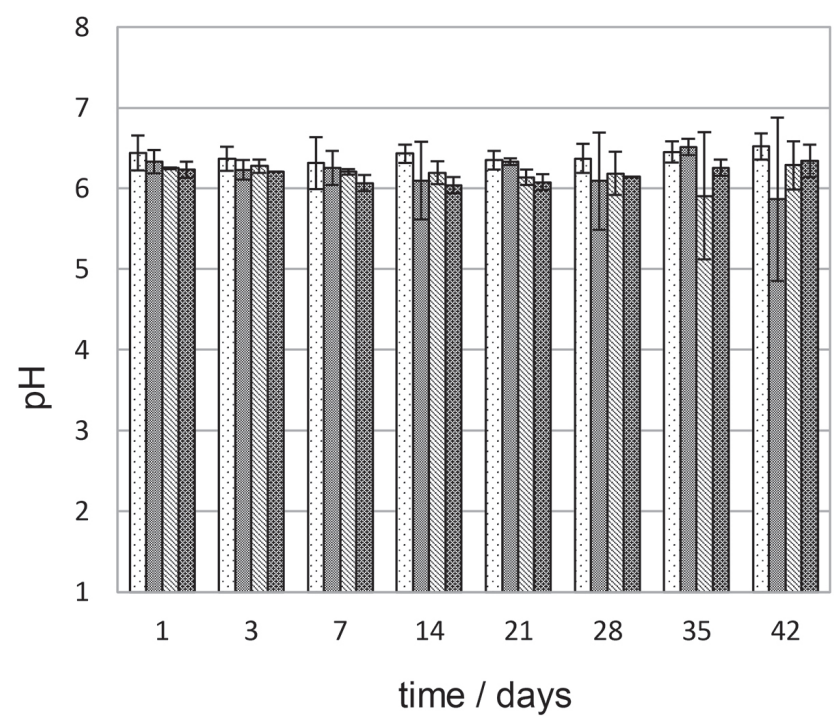

$\square$ NLC with active
at $4{ }^{\circ} \mathrm{C}$

$\square$ NLC without active at $4{ }^{\circ} \mathrm{C}$

$\mathbb{N L C}$ with active at room temperature

圈 NLC without active at room temperature

Figure 3. $\mathrm{pH}$ of the NLCs formulations stored at $4{ }^{\circ} \mathrm{C}$ and room temperature as a function of time. The statistical difference was $p>0.05$ in relation to the starting point ( 1 day) to NLC at room temperature and stored at $4{ }^{\circ} \mathrm{C}$ with and without active. It was analyzed through ANOVA with post-analysis using the Dunnett's test. Results presented as the mean and standard deviation of three independent experiments $(\mathrm{n}=3)$.

\section{Studies of interaction of nanoparticles and human skin}

Having characterized the NLC formulation and demonstrated that they are stable for almost 2 months, the NCLs interactions with human skin were investigated. Human skin was mounted in a Franz diffusion cell and exposed to the treatments. Figure 4 shows the control where the cross-section of human skin was exposed to the rhodamine-PE. After $1 \mathrm{~h}$, rhodamine is distributed on top of the skin section, mostly on the stratum corneum, showing a negligible signal inside the follicles and no indication of permeation into the dermis. Conversely, cross-sections of human skin exposed to the NLC formulation showed a distinct pattern (Figure 5). The fluorescence distribution indicates that NLC formulation accumulates less on the top of the skin and more into the hair follicles. Additionally, fluorescence was observed in the dermis, indicating NLC permeation into the dermis. ${ }^{32}$ Obtained data might be indicative of the main route for the nanoparticle dermal penetration through the follicle. This finding agrees with Abdel-Hafez et al. ${ }^{33}$ These authors have shown that the appendageal route is the main route of penetration of lipid nanoparticles, also demonstrating that being inside the hair follicles is crucial for drug diffusion to deep layers of the skin. 


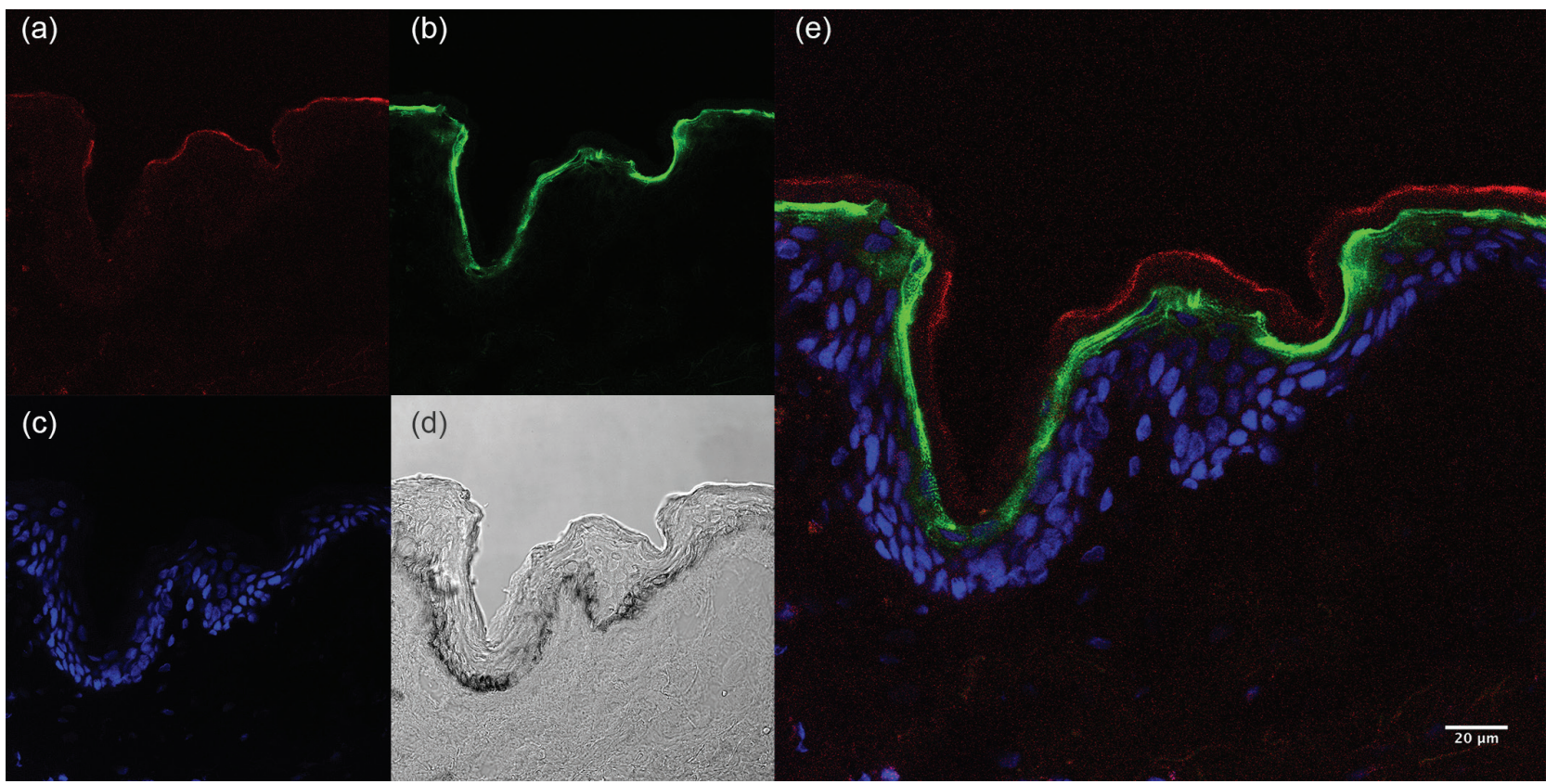

Figure 4. Representative confocal images of the cross-section of human skin exposed to the rhodamine-PE. (a) Rhodamine-PE (red); (b) actin labeled with Alexa Fluor 488 phalloidin (green); (c) nuclei labeled TO-PRO-3 (blue); (d) bright field; (e) overlay (merge) of (a), (b) and (c).

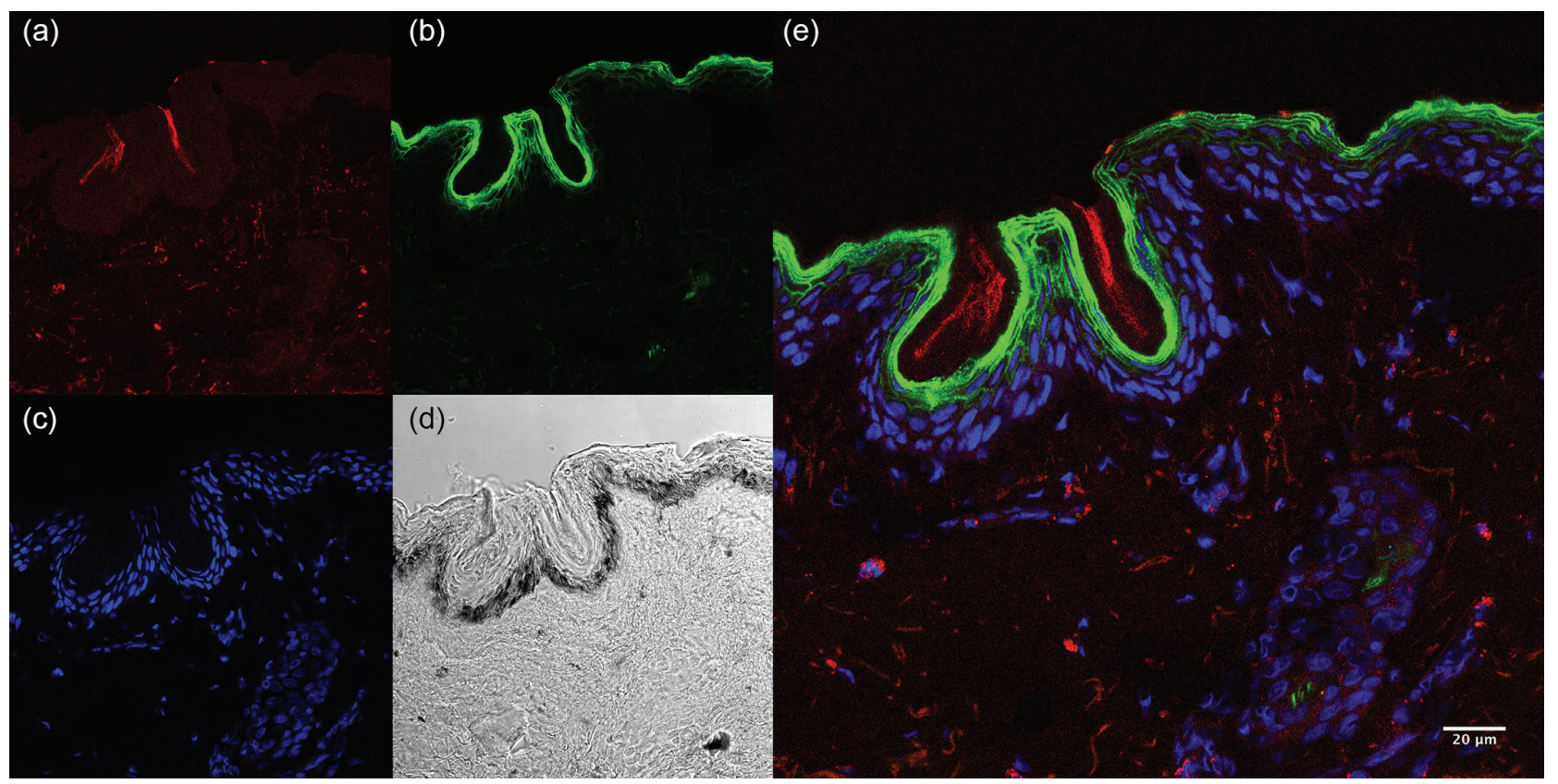

Figure 5. Representative confocal images of the cross-section of human skin exposed to the labeled NLC formulation: (a) NLCs labeled with rhodamine (red); (b) actin labeled with Alexa Fluor 488 phalloidin (green); (c) nucleus labeled TO-PRO-3 (blue); (d) bright field; (e) overlay (merge) of (a), (b) and (c).

\section{Conclusions}

NLCs with average diameters of $96 \pm 15 \mathrm{~nm}$ and the polydispersity index of $0.3 \pm 0.1$ were obtained, which is a significant result given the goal to improve the skin penetration of the 17- $\alpha$-estradiol. Also, high levels of entrapment $(99.6 \pm 0.3 \%)$ and high stability at the roomtemperature point to great encapsulation of $17-\alpha$-estradiol in NLCs. Confocal microscopy results showed that the NLC formulation efficiently penetrated and accumulated into the hair follicle and partially permeated through the dermis. The high levels of entrapment and the accumulation into the hair follicle could improve the 17- $\alpha$-estradiol efficiency. Knowing that $17-\alpha$-estradiol inhibits the $5 \alpha$-reductase located mainly in the dermal papilla cells at the base of the hair follicle, ${ }^{34}$ obtained NLCs could provide selective and efficient treatment for alopecia. 


\section{Acknowledgments}

Support from INOMAT (MCTI/CNPq), Brazilian Network on Nanotechnology (MCTI/CNPq), NanoBioss (MCTI) and FAPESP are acknowledged. We also appreciated the initial collaboration and suggestions from Dr P. D. Marcato, during her postdoctoral studies in our institution.

\section{References}

1. Hosking, A. M.; Juhasz, M.; Mesinkovska, N. A.; Skin Appendage Disord. 2019, 5, 72.

2. Wojciechowska-Zdrojowy, M.; Jankowska-Konsur, A.; EMJ, Dermatol. 2018, 6, 100.

3. Wiggins, S.; Moore-Millar, K.; Thomson, A.; Body Image 2014, 11,156 .

4. Motofei, I. G.; Rowland, D. L.; Tampa, M.; Sarbu, M. I.; Mitran, M. I.; Mitran, C. I.; Stoian, A. P.; Diaconu, C. C.; Paunica, D. S.; Georgescu, S. R.; J. Dermatol. Treat. 2019, DOI:10.1080/ 09546634.2019.1595507.

5. Hatema, S.; Nasr, M.; Moftah, N. H.; Ragai, M. H.; Geneidi, A. S.; Elkheshen, S. A.; Expert Opinion Drug Deliv. 2018, 15, 927.

6. Lane, M. E.; J. Microencapsulation 2011, 28, 709.

7. Guilherme, V.A.; Ribeiro, L. N. M.; Tofoli, G. R.; Franz-Montan, M.; de Paula, E.; de Jesus, M. B.; Curr. Pharm. Des. 2017, 23, 6659.

8. Singh, A. P.; Biswas, A.; Shukla, A.; Maiti, P.; Signal Transduction Targeted Ther. 2019, 4, 33.

9. Rosen, J.; Landriscina, A.; Friedman, A. J.; Cosmetics 2015, 2, 211.

10. Souto, E. B.; Müller, R. H.; Int. J. Cosmet. Sci. 2008, 30, 157.

11. Gomes, M. J.; Martins, S.; Ferreira, D.; Segundo, M. A.; Reis, S.; Int. J. Nanomed. 2014, 9, 1231.

12. Lin, Y. K.; Al-Suwayeh, S. A.; Leu, Y. L.; Shen, F. M.; Fang, J. Y.; Pharm. Res. 2012, 30, 435.

13. Ghasemiyeh, P.; Azadi, A.; Daneshamouz, S.; Heidari, R.; Azarpira, N.; Mohammadi-Samani, S.; Pharm. Dev. Technol. 2019, 24, 812 .

14. Kim, J. H.; Lee, S. Y.; Lee, H. J.; Yoon, N. Y.; Lee, W. S.; Ann. Dermatol. 2012, 24, 295.

15. Huh, C. H. In Practical Aspects of Hair Transplantation in Asians, $1^{\text {st }}$ ed.; Springer: Tokyo, 2018, p. 643.
16. Ruksiriwanich, W.; Manosroi, J.; Abe, M.; Manosroi, W.; Manosroi, A.; J. Supercrit. Fluids 2011, 59, 61.

17. Shen, Y. L.; Li, X. Q.; Pan, R. R.; Yue, W.; Zhang, L. J.; Zhang, H.; Curr. Pharm. Des. 2018, 24, 3090.

18. de Melo, N. F. S.; Grillo, R; Guilherme, V. A.; de Araujo, D. R.; de Paula, E.; Rosa, A. H.; Fraceto, L. F.; Pharm. Res. 2011, $28,1984$.

19. Gatti, R; Gioia, M. G.; Di Pietra, A. M.; Cavrini, V.; J. Pharm. Biomed. Anal. 1998, 18, 187.

20. Schneider, C. A.; Rasband, W. S.; Eliceiri, K. W.; Nat. Methods 2012, 9, 671 .

21. Lin, P. J. C.; Tam, Y. Y. C.; Hafez, I.; Sandhu, A.; Chen, S.; Ciufolini, M. A.; Nabi, I. R.; Cullis, P. R.; Nanomedicine 2013, 9, 233.

22. Adib, Z. M.; Ghanbarzadeh, S.; Kouhsoltani, M.; Khosroshahi, A. Y.; Hamishehkar, H.; Adv. Pharm. Bull. 2016, 6, 31.

23. Parmar, H. G.; Smolkova, I. S.; Kazantseva, N. E.; Babayan, V.; Pastorek, M.; Pizurova, N.; IEEE Trans. Magn. 2014, 50, 5200504.

24. Septiyanti, M.; Mulyana, M. R.; Putri, R.; Meliana, Y.; Am. J. Phys. Appl. 2019, 7, 14.

25. Kourniatis, L. R.; Spinelli, L. S.; Mansur, C. R. E.; Quim. Nova 2010, 33, 295.

26. Gillet, A.; Compère, P.; Lecomte, F.; Hubert, P.; Ducat, E.; Evrard, B.; Piel, G.; Int. J. Pharm. 2011, 411, 223.

27. Pivetta, T. P.; Simões, S.; Araújo, M. M.; Carvalho, T.; Arruda, C.; Marcato, P. D.; Colloids Surf., B 2018, 164, 281.

28. Rigon, R. B.; Gonçalez, M. L.; Severino, P.; Alves, D. A.; Santana, M. H.; Souto, E. B.; Chorilli, M.; Colloids Surf., B 2018, 171, 501.

29. Souto, E. B.; Winssing, S. A.; Barbosa, C. M.; Müller, R. H.; Int. J. Pharm. 2004, 278, 71.

30. Chirio, D.; Peira, E.; Dianzani, C.; Muntoni, E.; Gigliotti, L. C.; Ferrara, B.; Gallarate, M.; Nanomaterials 2019, 9, 230.

31. Choi, K. O.; Aditya, N. P.; Ko, S.; Food Chem. 2014, 147, 239.

32. Tomoda, K.; Watanabe, A.; Suzuki, K.; Inagi, T.; Terada, H.; Makino, K.; Colloids Surf., B 2012, 97, 84.

33. Abdel-Hafez, S. M.; Hathout, R. M.; Sammour, O. A.; Int. J. Biol. Macromol. 2018, 108, 753.

34. Inui, S.; Itami, S.; J. Dermatol. Sci. 2011, 61, 1.

Submitted: October 28, 2019

Published online: February 4, 2020 